\title{
Simplified WDM Optical Beamforming Network for Large Antenna Arrays
}

\author{
Borja Vidal, Teresa Mengual, Cristina Ibáñez-López, Javier Martí, Iain McKenzie, Emilio Vez, Javier Santamaría, \\ Francesc Dalmases, and Lluis Jofre
}

\begin{abstract}
A novel approach to simplify optical beamforming networks based on wavelength-division-multiplexing (WDM) optical sources and a dispersive medium is proposed. The technique relies on the combination of dispersive and nondispersive time delays to allow the reuse of optical carriers. Thus, the number of required optical sources, which is one of the main limitations of WDM-based beamforming architectures, can be drastically reduced. The approach eases the implementation of these architectures in large antenna arrays, reducing the cost, size, and complexity of the beamformer. Preliminary experimental results are provided to validate the concept.
\end{abstract}

Index Terms-Fiber dispersion, microwave photonics, optical beamforming, wavelength-division multiplexing (WDM).

\section{INTRODUCTION}

$\mathbf{T}$ HE USE of optics to process microwave and radio-frequency (RF) signals [1] allows the exploitation of the advantages of optical components: low loss, large bandwidth, low weight, and immunity to electromagnetic interference. Several applications have been proposed such as optical beamforming for antenna arrays, photonic microwave filters and photonic analog-to-digital converters. Optical beamforming is very attractive due to the capability of optical technology to avoid beam squint (i.e., to provide true time delay), a problem that arises when a large relative bandwidth and/or large antenna arrays are used. Many different optical beamforming architectures have been proposed so far [2]-[8] using a wide variety of implementations. Some of them are based on wavelength-division-multiplexing (WDM) technology (i.e., multiple simultaneous optical sources at different wavelengths) and a dispersive medium to time-delay the microwave signal modulated over the optical carriers. WDM-based optical beamformers provide scalability and simplify the architecture by using a set of optical carriers and implementing the time delays by means of chromatic dispersion. In addition, these WDM architectures benefit from using low-cost high-performance components available for fiber-optic communication networks.

\footnotetext{
Manuscript received November 28, 2005; revised February 17, 2006. This work was supported by the European Space Agency through the OBEFONE Project.

B. Vidal, T. Mengual, C. Ibáñez-López, and J. Martí are with the Nanophotonics Technology Center, Universidad Politécnica de Valencia, Valencia 46022, Spain (e-mail: bvidal@ieee.org).

I. McKenzie is with the European Space Agency, European Space Research and Technology Centre, 2200 AG Noordwijk, The Netherlands.

E. Vez and J. Santamaría are with SENER, Ingeniería y Sistemas, Aerospace Division, 28760 Tres Cantos, Madrid, Spain.

F. Dalmases and L. Jofre are with the Universitat Politècnica de Catalunya, 08034 Barcelona, Spain.

Digital Object Identifier 10.1109/LPT.2006.874708
}

Although continuous progress is done in the integration of optical single and multiple-carrier sources, the need of as many optical carriers as antenna elements makes difficult the implementation of optical beamformers for large antenna arrays with many elements. It would be very useful to develop techniques that allow the optical control of large antenna arrays and, thus, fully exploit the advantages of photonic technology in real applications.

Recently, a technique to reduce the number of optical sources in WDM photonic microwave filters has been proposed [9]. It allows the reduction of the number of optical sources by means of the combination of dispersive and nondispersive time delays to make more efficient use of the WDM parallelism. In this letter, the feasibility of the technique to reduce the number of optical sources in WDM-based optical beamforming networks is proposed and demonstrated.

\section{PRINCIPLE OF OPERATION}

In optical beamforming networks based on several optical carriers and a dispersive medium a correspondence is established between each optical carrier and each antenna element [5]-[7]. However, it is feasible to reduce the number of optical sources of the architecture if the optical carriers are reused by means of the combination of dispersive and nondispersive time delays. After the dispersive medium (i.e., after chromatic dispersion has introduced a time delay between optical carriers $\Delta \tau)$, the WDM signal is split in several branches, using variable nondispersive optical delay lines [(ODLs) which are wavelength-independent and affect equally to all the carriers within a set] to introduce an absolute time delay $(\Delta T)$ and time interleave the different sets of carriers.

Thus, a progressive time-delay profile is obtained as represented in Fig. 1. The absolute time delay at branch $k, \Delta T_{k}$, should be equal to $(k-1) \Delta \tau / M$, where $M$ is the total number of branches and $k$ is an integer between 1 and $M$.

As can be seen in Fig. 2, a set of $N$ optical carriers is amplitude-modulated and driven to a dispersive medium. Chromatic dispersion will introduce a relative time delay between optical carriers $\Delta \tau=D \cdot L \cdot \Delta \lambda$, where $D$ is the fiber dispersion parameter, $L$ is the fiber length, and $\Delta \lambda$ is the wavelength spacing between optical carriers. Unlike previous proposals, the $N$ optical carriers are equally split into $M$ branches. Thus, a set of amplitude-modulated optical carriers with a constant time delay between them is launched to each branch. Nondispersive variable ODLs are used to time-interleave these sets of optical carriers. Then, each optical carrier is demultiplexed and routed to one photodiode, which feeds one antenna element. 


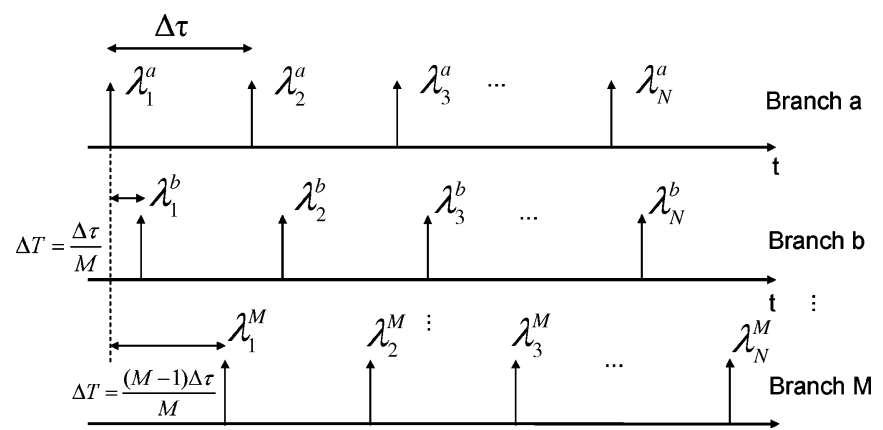

Fig. 1. Combination of dispersive $(\Delta \tau)$ and nondispersive $(\Delta T)$ time delays to obtain the interleaving of time-delayed samples of the electrical signal.

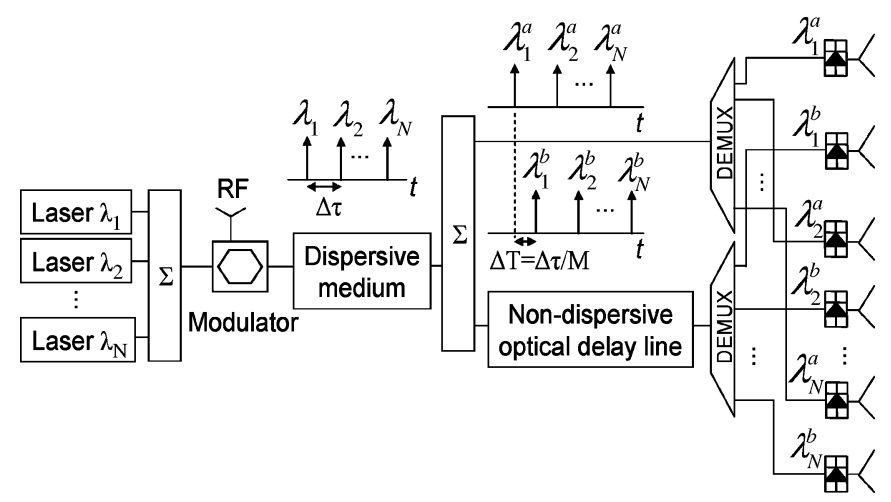

Fig. 2. Concept of the simplified beamforming architecture.

There are two ways to obtain a progressive time delay between antenna elements: it is possible to time-interleave, by means of nondispersive time delays, the sets of dispersively time-delayed optical carriers (as schematically shown in Fig. 1, using $\left.\Delta T_{k}=(k-1) \Delta \tau / M\right)$ or to delay one whole set after another (i.e., $\left.\Delta T_{k}=(k-1) N \Delta \tau\right)$. The former (Fig. 1) reduces the time delay introduced by the delay lines and, therefore, this is preferred.

Fig. 2 shows the concept of the simplified beamforming architecture. Using $N$ optical sources, $M-1$ ODLs, and $M$ demultiplexers, the number of antenna elements controlled by the beamforming network is $N \times M$ whereas the number of components (optical sources and demultiplexers) is $N+2 M-1$. In addition to these components, other devices are needed, for example, one external modulator or a dispersive medium, but these do not scale with the number of antenna elements. The number of photodiodes also scales with the size of the antenna array but their number is the same in this and previous architectures [5]-[7] since they are needed to convert the signals from the optical to the electrical domain. Fig. 3 compares the number of components needed using the proposed technique with previous WDM beamforming architectures [5]-[7]. Fig. 3 shows how the number of components is highly reduced using this technique when the number of antenna elements is large.

Since the reduction in the number of components is based on the reuse of each optical carrier to feed several antenna elements, the optical power of each carrier is divided by $M$. It is required that the optical power of each optical carrier has to be increased by $10 \cdot \log _{10}(M) \mathrm{dB}$ plus the coupler excess loss. This can limit the maximum value of $M$. This upper limit depends on

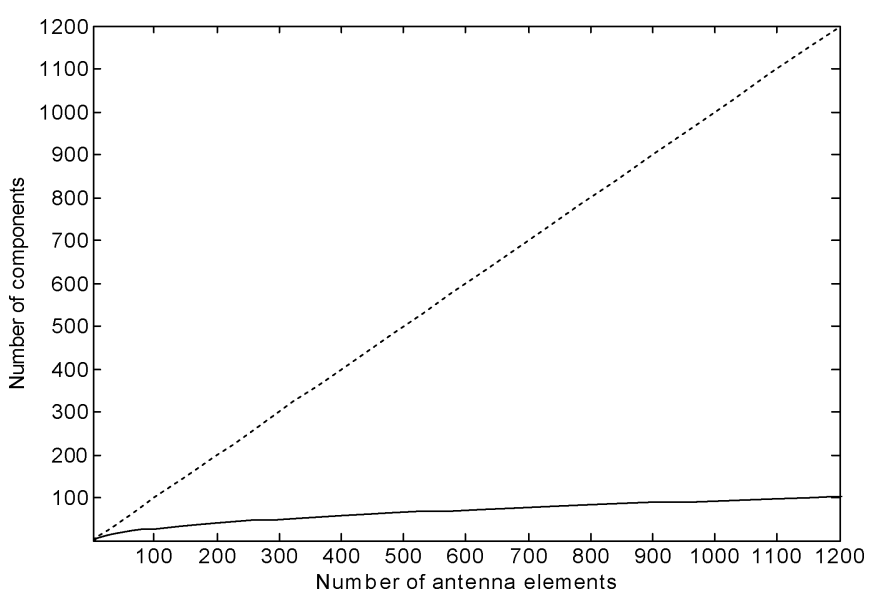

Fig. 3. Comparison of the number of components (optical sources and demultiplexers) between the proposed technique (solid) and previous WDM beamforming networks (dashed) for $N=M$ with values from 1 to 35 .

the required power budget of the system but if the maximum additional insertion loss relative to an architecture such as [6] and [7] were, for instance, $13 \mathrm{~dB}$, the maximum $M$ would be around 20. However, this limit can be increased using lasers with higher output power or using optical amplifiers before the demuxes. It is easier than using $N \times M$ optical sources. In addition, the technique also slightly increases the complexity of the control subsystem.

There are two extreme cases: 1) only dispersive time delays ( $N=K, K$ being the total number of antenna elements, and $M=1$ ) which is equivalent to previous schemes such as [4]; and 2) only nondispersive time delays ( $N=1$ and $M=K$ ) which is equivalent to previous schemes such as [6]. Therefore, the technique proposed here can be seen as a general case of these two previous approaches. The optimum design point that maximizes the number of antenna elements and minimizes the number of components is $N \approx M$. In this case, the additional power required in each optical carrier would be $\sim 10 \log _{10}(\sqrt{K})$.

This technique is independent of the WDM source used, thus, the spectrum slicing of broadband sources could be employed to further reduce the beamformer cost. As usual in WDM beamforming architectures, to steer the beam, the dispersive time delay has to be changed (for example, by changing the total length of fiber using optical switches [6] or by changing the wavelength spacing between optical carriers by means of tunable optical sources [7]). At the same time that the dispersive time delay is changed, the time delay of the variable ODLs have to be properly modified to maintain the time-delay profile. This technique is intended to provide a variable time delay between antenna elements but the same for every element.

The technique can be used for generating discrete and continuous beamsteering depending on the dispersive time-delay generation method and the kind of ODL used (i.e., ODLs with discrete or continuous time-delay tuning steps).

\section{EXPERIMENTAL SETUP}

Fig. 4 shows the block diagram of the experimental setup used to demonstrate the principle of operation. The time delay 


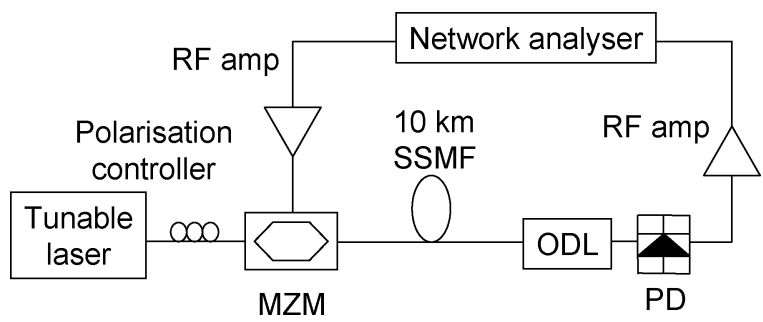

Fig. 4. Block diagram of the experimental setup used to show the feasibility of the principle. MZM stands for Mach-Zehnder modulator, and SSMF for standard single-mode fiber.

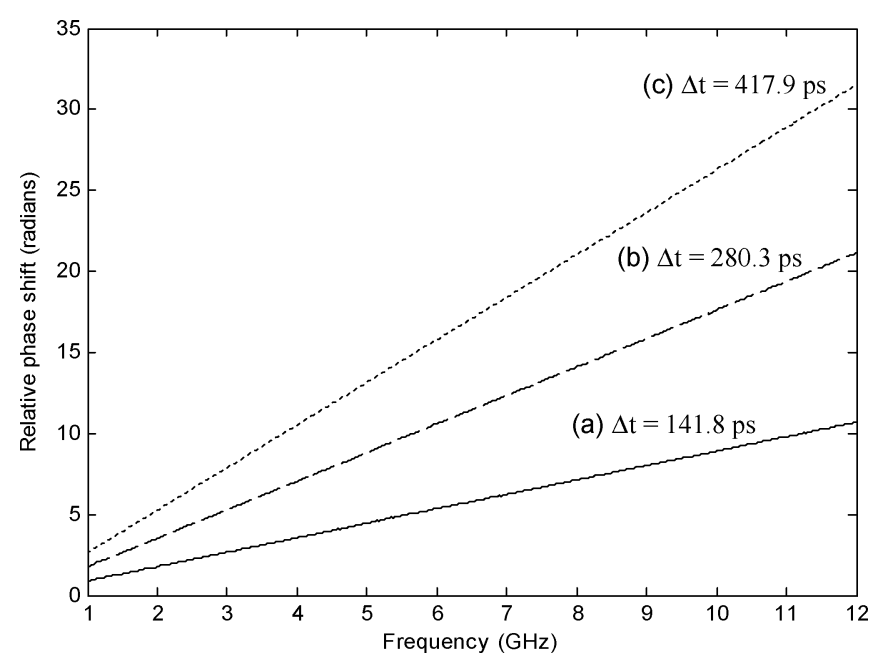

Fig. 5. Relative RF phase shift versus frequency relative to the reference (optical carrier at $\lambda=1550 \mathrm{~nm}$ and ODL $=0 \mathrm{ps}$ ) for three cases: (a) $\lambda=1550 \mathrm{~nm}$, ODL $=140 \mathrm{ps}$; (b) $\lambda=1551.6 \mathrm{~nm}$, ODL $=0 \mathrm{ps}$; and (c) $\lambda=1551.6 \mathrm{~nm}$, ODL $=140 \mathrm{ps}$.

introduced over optical carriers of different wavelength by the combination of a dispersive medium and a nondispersive ODL has been measured using a single tunable source, i.e., the time delays of the different optical carriers are not measured simultaneously. To measure the time delay, a tunable laser is amplitude-modulated by the signal generated in an electrical network analyzer (port \#1). A wavelength-dependent time delay is introduced by the chromatic dispersion of a coil of $10 \mathrm{~km}$ of standard single-mode fiber. The nondispersive time delay is generated using a commercial tunable ODL. Then, the signal is photodetected, amplified, and launched to the electrical network analyzer port \#2. The time delay is measured from the slope of the phase response of parameter $S_{21}$ versus frequency.

Fig. 5 shows the time-delay measurements obtained with the experimental setup of Fig. 4 for two optical carriers with a wavelength spacing of $1.6 \mathrm{~nm}\left(\lambda_{1}=1550 \mathrm{~nm}\right.$ and $\left.\lambda_{2}=1551.6 \mathrm{~nm}\right)$ and introducing two time delays with the ODL $(\Delta T=0$ and
$\Delta T=140 \mathrm{ps})$. The theoretical time delay introduced by the dispersive medium is $\Delta \tau=D \cdot L \cdot \Delta \lambda=17.5 \cdot 10 \cdot 1.6=280 \mathrm{ps}$. To interleave the two sets of optical carriers delayed by chromatic dispersion, the ODL has to introduce a time delay equal to $\Delta \tau / 2=140 \mathrm{ps}$ to obtain progressive equally spaced time delays. Thus, it is possible to obtain four different theoretical time delays: $0,140,280$, and 420 ps. Fig. 5 shows the phase shift versus frequency for three measurements relative to the minimum time delay ( $\left.\lambda_{1}=1550 \mathrm{~nm}, \Delta T=0 \mathrm{ps}\right)$. From their slope, the time delay of each one can be obtained.

As can be seen from the measurements of Fig. 5, which show a good agreement with theory, it is possible to combine dispersive and nondispersive time delays to generate progressive time delays and reduce the number of components.

\section{CONCLUSION}

In this letter, it has been shown how WDM optical beamforming architectures can be simplified combining dispersive and nondispersive time delays to allow the use of photonic beamforming techniques in large antenna arrays. The number of optical sources of the beamformer, as well as its total cost, can be highly reduced using this technique. This is done at the expense of higher insertion loss that can limit the maximum $M$ although it can be compensated using amplifiers. Experimental results validating the feasibility of the technique have been provided.

\section{REFERENCES}

[1] A. J. Seeds, "Microwave Photonics," IEEE Trans. Microw. Theory Tech., vol. 50, no. 3, pp. 877-887, Mar. 2002.

[2] H. Zmuda and E. H. Toughlian, Photonic Aspects of Modern Radar. Norwood, MA: Artech House, 1994.

[3] W. Ng, A. Walston, G. Tangonan, J. Newberg, J. J. Lee, and N. Berstein, "The first demonstration of an optically steered microwave phased array antenna using true-time delay," J. Lightw. Technol., vol. 9, no. 9, pp. 1124-1131, Sep. 1991.

[4] R. A. Soref, "Programmable time-delay devices," Appl. Opt., vol. 23, no. 21, pp. 3736-3737, Nov. 1984.

[5] O. Raz, R. Rotman, Y. Dazinger, and M. Tur, "Implementation of a photonic true-time delay using high-order-mode dispersion compensating fibers," IEEE Photon. Technol. Lett., vol. 16, no. 5, pp. 1367-1369, May 2004.

[6] D. T. K. Tong and M. C. Wu, "A novel multiwavelength optically controlled phased array antenna with a programmable dispersion matrix," IEEE Photon. Technol. Lett., vol. 8, no. 6, pp. 812-814, Jun. 1996.

[7] J. L. Corral, J. Marti, S. Regidor, J. M. Foster, R. Laming, and M. J. Cole, "Continuously variable true time-delay optical feeder for phased-array antenna employing chirped fiber gratings," IEEE Trans. Microw. Theory Tech., vol. 45, no. 8, pp. 1531-1536, Aug. 1997.

[8] S. Granieri, M. Jaeger, and A. Siahmakoun, "Multiple-beam fiber-optic beamformer with binary array of delay lines," J. Lightw. Technol., vol. 21, no. 12, pp. 3262-3272, Dec. 2003.

[9] B. Vidal, V. Polo, J. L. Corral, and J. Martí, "Efficient architecture for WDM photonic microwave filters," IEEE Photon. Technol. Lett., vol. 16, no. 1, pp. 257-259, Jan. 2004. 